\title{
Twin study identifies an association between exposure to a solvent and risk of Parkinson disease
}

A solvent that is used in a wide range of household products and is present as an environmental contaminant in groundwater could increase the risk of developing Parkinson disease (PD), according to a twin study.

A small number of case reports had previously suggested a link between exposure to the solvent trichloroethylene (TCE) and PD. In the current study, the researchers contacted members of the National Academy of Science/National Research Council World War II Veteran Twins registry-a large, all-male twin cohort in the USA.

Individuals with suspected PD and their twins were evaluated by a movement disorder specialist in order to identify twin pairs discordant for PD, which would control for genetic and many lifestyle and demographic factors. These pairs then completed detailed questionnaires about job tasks they had undertaken from the age of 10 years. "This approach helped to mitigate recall bias, because subjects were asked to report job tasks rather than specific compounds," explains Sam Goldman, who was the lead author of the paper. Occupational history data were collected from 99 twin pairs, and exposure to six selected solvents, including TCE, was inferred by an industrial hygienist or occupational medicine physician, who were blinded to case status.

The results showed that individuals who were ever exposed to TCE had a significantly increased risk of developing $\mathrm{PD}(\mathrm{OR}=6.1 ; P=0.034)$. A trend towards significance was also observed for the solvents tetrachloroethylene (also known as perchloroethylene [PERC]) and carbon tetrachloride. Exposure to the solvents toluene, xylene and $n$-hexane was not associated with an increased risk of developing PD.

"Both TCE and PERC are metabolized to a compound known as TaClo, which is a potent mitochondrial complex 1 inhibitor," says Goldman. Inhibition of this mitochondrial protein may have a pathogenic role in $\mathrm{PD}$, suggesting a possible mechanistic link between these solvents and the disease.

The results on TCE are consistent with studies in a TCE-induced rat model of $\mathrm{PD}$, which recapitulates many of the pathological hallmarks of the disease, including loss of dopaminergic neurons and accumulation of $\alpha$-synuclein in the substantia nigra.

This study could provide important clues about environmental factors in PD but, as Goldman cautions, "a single association study does not prove causality." In future work, his group is hoping to replicate the results in independent study populations.

Katie Kingwell

Original article Goldman, S. M. et al. Solvent exposures and Parkinson disease risk in twins. Ann. Neurol. doi:10.1002/ana.22629 